$\underline{\text { Preprint typeset in JHEP style. - HYPER VERSION }}$

$\mathrm{UW} / \mathrm{PT}-00 / 01$

IASSNS-HEP-00/46

\title{
Suppressing Flavor Anarchy
}

\author{
Ann E. Nelson \\ Department of Physics, Box 1560, University of Washington, \\ Seattle, WA 98195-1560, USA
}

\section{Matthew J. Strassler}

School of Natural Sciences, Institute for Advanced Study, Princeton, NJ 08540, USA

\begin{abstract}
We present a new mechanism, which does not require any flavor symmetry, to explain the small Yukawa couplings and CKM mixing angles. The Yukawa matrices are assumed to be random at short distances and the hierarchical structure is generated in the infrared by renormalization group flow. The generic qualitative predictions of this mechanism are in good agreement with observation. We give several simple examples in supersymmetric theories. We show that our mechanism can also ameliorate the supersymmetric flavor problem, and make predictions for the superpartner mass spectrum. The mechanism is fully consistent with grand unification, and in $S U(5)$-based models of neutrino mass, predicts a large mixing angle for $\nu_{\mu} \leftrightarrow \nu_{\tau}$ oscillations.
\end{abstract}




\section{Introduction}

The quark and lepton masses and mixing angles exhibit a degree of structure and regularity which is puzzling, as in the Standard Model and typical extensions these are determined by arbitrary Yukawa coupling matrices. In particular, the three generations of quarks and leptons have quite disparate masses spanning more than five orders of magnitude, with rather small mixing angles between the different generations, and with the largest mixing between adjacent generations.

In this paper we show how such a pattern can arise as the result of renormalization group running, when some of the quarks and leptons acquire substantial anomalous dimensions that persist over a large range of energy scales. We give supersymmetric examples where such anomalous dimensions are the result of superpotential couplings of the light quarks and leptons to a superconformal sector. The sizes of the resulting Yukawa couplings are set by an accidental and approximate $U(1)_{R}$ symmetry, whose presence is a consequence of the approximate superconformal symmetry in the strong-coupling sector. The resulting predictions resemble those of successful flavor models with horizontal abelian symmetries and with all standard model fermions carrying charges with the same sign [1, 2, 3, 4. In our scenario, the R-symmetry which sets the charges arises dynamically and accidentally, and unitarity demands all matter fields have positive charges; thus these features do not have to be imposed by hand.

We also examine the soft supersymmetry breaking terms in these models. The trilinear scalar interactions associated with superpartners of the light fermions are dramatically reduced, and have similar texture to the Yukawa matrices. They are not, however, necessarily proportional to Yukawa couplings, leading to interesting flavor violating signatures. In a large class of models, the renormalized masses of the scalar superpartners of the light fermions become nearly degenerate, suppressing the supersymmetric contribution to flavor-changing processes.

\section{How a CFT can produce the flavor hierarchy}

Typically, dimensionless coupling constants such as Yukawa couplings have logarithmic energy dependence, and so renormalization group flow cannot account for very large ratios of dimensionless couplings, even in a strongly-coupled theory. This is because the effective theory is describable in terms of weakly-coupled degrees of freedom at most energies and is only truly strongly coupled near one particular energy scale (although the weakly-coupled degrees of freedom may be different in the ultraviolet and infrared). However it was realized some time ago [5] that in a theory with a nearly scale invariant gauge coupling the Yukawa couplings can run like a power of the renormalization group scale $\mu$, and that a large Yukawa hierarchy could result from renormalization group running.

Many field theories which are free in the ultraviolet will flow to a Conformal Fixed Point (CFT) in the infrared, such as the simple example discussed in ref. [6]. A prototypical 
supersymmetric example is supersymmetric QCD with $\frac{3}{2} N_{c}<N_{f}<3 N_{c}$ [7]. In this section, we examine the effect of coupling the standard model to an approximate supersymmetric CFT. At the CFT, the operators of the free theory develop anomalous dimensions of order one. In many supersymmetric cases, these anomalous dimensions are computable even at strong coupling, using superconformal symmetry. The large anomalous dimensions cause rapid running of the Yukawa couplings. Such anomalous Yukawa coupling scaling leads to a simple explanation of the hierarchy of quark and lepton Yukawa couplings. As we will show in section 3, the generic predictions of this mechanism, resembling the most successful models of [1, 2, 3, 迎, give qualitative relationships between mass ratios and mixing angles which are in striking agreement with reality.

\subsection{Effect of a Conformal Field Theory on a Weakly Coupled Sector}

The effect of coupling a superfield $X$ of the MSSM to a CFT via the superpotential was considered in Sec. (2.1) of [8]. We summarize the results of that section here.

Consider an $\mathcal{N}=1$ supersymmetric gauge theory with gauge group $G$, charged matter $Q$, neutral matter $X$, and a superpotential $W(Q, X)$. Suppose that this theory becomes conformal in the infrared. Unitarity then requires that all gauge-invariant operators $\mathcal{O}$ (except the identity operator) have dimension $\operatorname{dim} \mathcal{O}$ greater than or equal to one; if $\operatorname{dim} \mathcal{O}=1$, then the operator satisfies the Klein-Gordon equation $\partial^{2} \mathcal{O}=0$ [9, 10]. By assumption $Q$ is not gauge invariant, although there will be gauge-invariant chiral operators $\mathcal{O}(Q)$ which are multilinear in $Q$. This means $Q$ may have a negative anomalous dimension. By contrast, $X$ is gauge invariant, so $\operatorname{dim} X \geq 1$ and its anomalous dimension $\gamma_{X}=2[\operatorname{dim} X-1] \geq 0$; if $\gamma_{X}=0$ then $X$ must decouple in the infrared from the remainder of the theory.

What is the effect of this positive anomalous dimension? First, recall that at any superconformal fixed point there is an anomaly-free $U(1)$ R-symmetry; the dimension of a chiral operator $\mathcal{O}$ is determined by its charge under the R-symmetry, $\operatorname{dim}(\mathcal{O})=\frac{3}{2} R_{\mathcal{O}}$. It follows that if $\mathcal{O}$ and $\mathcal{O}^{\prime}$ are chiral then $\operatorname{dim}\left(\mathcal{O O}^{\prime}\right)=\operatorname{dim} \mathcal{O}+\operatorname{dim} \mathcal{O}^{\prime}$. Now let us consider a given term $c w$ in the superpotential $W$, where $w$ is an operator and $c$ is its coefficient. Note $\operatorname{dim} c+\operatorname{dim} w=\operatorname{dim} W=3$. The operator $w$ must contain $k \geq 0$ powers of neutral fields $X$ times a $G$-invariant chiral operator $\mathcal{O}$ built from the $Q$ fields. If $\mathcal{O}$ is not the unit operator, then $\operatorname{dim} \mathcal{O} \geq 1$, so $\operatorname{dim} w \geq k+1$; similarly, if $\mathcal{O}$ is the unit operator, $\operatorname{dim} w \geq k$.

The beta function for $c$ is simply

$$
\frac{\partial c}{\partial \ln \mu} \equiv \beta_{c}=c(\operatorname{dim} w-3)
$$

so if $\operatorname{dim} w>3$ in the infrared, $c$ will flow to zero and the term $c w$ will disappear from the superpotential of the CFT. ${ }^{1}$ Therefore, at or near such a conformal fixed point, almost all operators that can appear in the superpotential are irrelevant. The only marginal or relevant

\footnotetext{
${ }^{1}$ The possibility that relevant or marginal operators in the Kähler potential couple the fields $X$ to the CFT is not excluded by this argument. However, no examples of this phenomenon are known. We will assume such couplings do not occur in the CFTs that we discuss below.
} 
operators are of the form $\mathcal{O}(Q)$ (for $\operatorname{dim} \mathcal{O} \leq 3$, ) $X \mathcal{O}(Q)$, (for $\operatorname{dim} \mathcal{O}$ strictly less than 2,) or $X X$ (if $\operatorname{dim} X X \leq 3$.) Note that if $X \mathcal{O}(Q)$ is to appear in the superpotential of the CFT, the constraint $1<\operatorname{dim} \mathcal{O}<2$ generally implies that $0<\gamma_{X}<2$. (This last constraint can be avoided if $\mathcal{O}$ is redundant, that is, set equal to a total derivative by the equations of motion. In this case $\mathcal{O}$ is not primary and the relation between $\mathrm{R}$-charge and dimension is modified. However, the R-charge of $\mathcal{O}$ still determines the R-charge, and thereby the dimension, of $X$.)

In particular, consider what happens to a Yukawa coupling of the form $y X_{1} X_{2} X_{3}$, where the $X_{i}$ are all neutral under $G$. This has has beta function

$$
\beta_{y}=\frac{y}{2}\left(\gamma_{1}+\gamma_{2}+\gamma_{3}\right)
$$

where $\gamma_{i}$ is the anomalous mass dimension of the field $X_{i}$ at or near the conformal fixed point of $G$. As emphasized above, these anomalous dimensions must be positive, and so $y$ flows to smaller values in the infrared:

$$
y(\mu)=y\left(\mu_{0}\right) \times\left(\frac{\mu}{\mu_{0}}\right)^{\frac{1}{2}\left(\gamma_{1}+\gamma_{2}+\gamma_{3}\right)}=y\left(\mu_{0}\right) \times\left(\frac{\mu}{\mu_{0}}\right)^{\operatorname{dim}\left(X_{1} X_{2} X_{3}\right)-3} .
$$

Now suppose that the CFT in question has an exact $S U(3) \times S U(2) \times U(1)$ global symmetry, under which some of the $X$ and $Q$ fields are charged. If we weakly gauge this symmetry, with the standard model gauge couplings $g_{i}$, the effect on the CFT is small; both beta functions and anomalous dimensions change by of order $g_{i}^{2} / 16 \pi^{2}$. If the anomalous dimensions of the $X$ fields were already large for $g_{i}=0$, then the physics of the approximately conformal theory with non-zero $g_{i}$ is qualitatively and quantitatively similar to that of the CFT with $g_{i}=0$.

Thus, we consider the following scenario. In the effective theory below the scale of quantum gravity, we have gauge group $G \times S U(3) \times S U(2) \times U(1)$, with $G$ strongly coupled and the standard model relatively weakly coupled. If the quarks and leptons are coupled in the superpotential to the charged matter of $G$, conformal dynamics associated with $G$ will cause them to develop large anomalous dimensions. These in turn will cause their Yukawa couplings to the Higgs bosons to run to small values, as in Eq. (2.1).

More specifically, consider for example the leptons and their Yukawa couplings $y_{a i} L_{a} H_{d} E_{i}$, where $L_{a}$ and $E_{i}(i, a=1,2,3)$ are the doublet and singlet leptons of the three generations, and $H_{d}$ is the down-type Higgs doublet. Suppose a CFT caused $L_{a}$ and $E_{i}$ to develop anomalous dimensions $\gamma_{a}$ and $\gamma_{i}$ respectively. Let us imagine that all Yukawa couplings are of order one at the Planck or string scale $M_{0}$. Let us further imagine that the gauge coupling of $G$ and the Yukawa couplings involving $Q$ become conformal near $M_{0}$, and that the CFT decouples from the standard model (through dynamics we will discuss later) at a scale $\mu=M_{c}$. Define

$$
\epsilon_{a}^{L} \equiv\left(\frac{M_{c}}{M_{0}}\right)^{\frac{1}{2} \gamma_{a}} ; \epsilon_{i}^{\bar{E}} \equiv\left(\frac{M_{c}}{M_{0}}\right)^{\frac{1}{2} \gamma_{i}}
$$

Then, as in (2.1), the Yukawa couplings will run down to values

$$
y_{a i}\left(M_{c}\right) \sim \epsilon_{a}^{L} \epsilon_{i}^{\bar{E}}
$$


Below this scale the usual RG equations of the standard model will apply. Thus, if the $\gamma_{a}, \gamma_{i}$ have a hierarchy of order one, this hierarchy is exponentiated into a large lepton mass hierarchy. Since we want to obtain an electron Yukawa coupling of order $3 \times 10^{-6}$, and since $\gamma_{e}$ typically cannot be larger than 2 , we will typically need $M_{0} / M_{c} \sim 10^{2}-10^{8}$, depending on the details of the model. Note this can be much less than $M_{0} / M_{W}$, and can even be of order $M_{0} / M_{G U T}$ in some cases.

The lesson from the above paragraph is simple. Each quark or lepton $X$ coupled to the fields $Q$ in the superpotential will develop a large anomalous dimension $\gamma_{X}$, and an associated suppression factor

$$
\epsilon \equiv\left(\frac{M_{c}}{M_{0}}\right)^{\frac{1}{2} \gamma_{X}}=\sqrt{\frac{Z_{X}\left(M_{0}\right)}{Z_{X}\left(M_{c}\right)}},
$$

where $Z_{X}(\mu)$ is the Wilsonian wave-function renormalization factor for $X$ at the scale $\mu$. This means that a Yukawa coupling $y_{12} X_{1} X_{2} H$, where $H$ is a Higgs boson, will run down to

$$
y_{12}\left(M_{c}\right) \sim y_{12}\left(M_{0}\right) \epsilon_{1} \epsilon_{2} .
$$

where $\epsilon_{i}$ is the suppression factor for $X_{i}$.

Although this scenario does not lead to precise predictions, it does give order-of-magnitude relations. There are three possible sources of such relations. First, all supersymmetric CFTs have a special $U(1)$-R symmetry which determines anomalous dimensions for all chiral operators $\mathcal{O}$, through the relation $\operatorname{dim} \mathcal{O}=\frac{3}{2} R_{\mathcal{O}}$ [10, 11]. When (as is not always the case) this symmetry can be uniquely determined, $\gamma_{X}$ can be calculated (up to corrections of order standard model loop-factors) and so $\epsilon_{X}$ is known for all quarks and leptons as a function of $M_{c} / M_{0}$. Even if $M_{c}$ is a free parameter, there are still predictions, since when the $\gamma_{X}$ are known, there will be mass ratios in which $M_{c}$ cancels out.

Second, even if $\gamma_{X}$ is not known, it may be that there are GUT-type or other relations between the $\epsilon$ suppression factors for different standard model multiplets which reduces the number of parameters. For example, if an $S U(5)$ relation links the suppression factors of doublet quarks and up-type antiquarks, it leads to order-of-magnitude predictions for the mixing angles of the CKM matrix. Similar $S U(5)$ relations lead to predictions for lepton masses and neutrino oscillation parameters, as we will discuss in section 4 .

Finally, even if all the $\epsilon$ 's are left as free parameters, there are still some generic predictions for quark and lepton mixing angles, as will be discussed in greater detail in section 3. We will see that all such predictions either are satisfied in nature or have not yet been tested.

Note that to obtain large suppression factors does not require an exact CFT. A nearCFT with slow drift, or a flow from one approximate CFT to another, will imply anomalous dimensions for quarks and leptons which are not constants. This will make it difficult to compute the suppression factors $\epsilon_{i}$. However, this is a problem only for model builders and experimental verification, not one for nature. Obtaining a reasonable fermion mass spectrum only requires that anomalous dimensions be large, but not necessarily constant, over a significant range of energies. 


\subsection{Comments on the Origin of the Generational Hierarchy}

The reader may be concerned that we will need to impose flavor symmetries to ensure the generational hierarchy, and particularly to assure that the top quark does not develop a substantial anomalous dimension, which would drive its Yukawa coupling unacceptably small. Our scenario makes no such requirement. In particular, the model at the Planck scale has no notions of generational structure; all Yukawa couplings are of order one, and thus all mixings are large. Generational structure is an output of the model, not an input.

An essential property of $\mathcal{N}=1$ supersymmetric conformal field theories is that they always contain an anomaly-free $U(1)$ R-symmetry, under which all gauge-invariant operators carry a definite charge. This symmetry need not be present away from the conformal point; it may be violated by a host of irrelevant operators, and may exist only as an accidental symmetry in the infrared. At an approximate fixed point it of course will be only approximately conserved.

In the scenario discussed here, we have an approximate conformal fixed point, whose conformal invariance is weakly broken by the standard model gauge and Yukawa couplings. We therefore have an approximate $U(1)$ R-symmetry that the couplings of the model associated with the strong dynamics - those which would still be present if the conformal invariance were exact - must respect. Note that we need not impose that our theory have a $U(1)$ Rsymmetry in the ultraviolet; the dynamics will ensure that the theory develops an accidental approximate $U(1)$ in the regime where the theory is approximately conformal.

The existence of this $U(1)$ is essential to our mechanism. The standard model matter superfields are gauge-invariant operators with respect to the conformal sector. Consider, say, the three generations of lepton doublets $L_{i}^{(0)}$. In an exactly conformal regime, there must exist

three orthogonal linear combinations $L_{i}$ of the $L_{i}^{(0)}$ which carry definite and generally distinct R-charge assignments. Consequently, these three combinations cannot mix with one another and have distinct anomalous dimensions. This ensures that each standard model multiplet can be taken to have its own suppression factor. These statements remain approximately true when the conformal invariance is only approximate.

As illustration, suppose an exact CFT has operators $\mathcal{O}_{a}$ with R-charge $r_{a}$ and dimensions $d_{a}=\frac{3}{2} r_{a}$. A standard model field $X_{i}$ with R-charge $r_{i}$ can couple only to those operators with $r_{a}+r_{i}=2$. More precisely, if a term $X_{i} \mathcal{O}_{a}$ appears in the superpotential of a CFT, then the term $X_{i} \mathcal{O}_{b}$ cannot appear if $r_{a} \neq r_{b}$. Thus, suppose the operators with couplings to the lepton doublets $L_{i}^{(0)}$ have R-charges $r_{a}$ with $r_{1}<r_{2}<r_{3}<r_{4}<\ldots$. In this case, only one linear combination of the $L_{i}^{(0)}$ can couple to $O_{1}$. We may take this by definition to be $L_{1}$. In a similar way we may define $L_{2}$ to be the linear combination which couples to $\mathcal{O}_{2}$, and $L_{3}$ to be that which couples to $\mathcal{O}_{3}$. No linear combination will couple to $\mathcal{O}_{4}$ (unless a nongeneric cancellation has made one of the previous couplings zero.) Since $L_{1}, L_{2}$ and $L_{3}$ in this basis have different R-charges, they are orthogonal and do not mix; their kinetic terms are diagonal.

We may now ask how the theory approaches this fixed point, starting from an arbitrary 
Kähler potential and superpotential. The answer is that the initial condition doesn't matter, as long as it is reasonably generic. Whatever the starting point, the approach to the CFT will ensure that the low-energy theory can be written conveniently in a basis with diagonal kinetic terms and diagonal $X_{i} \mathcal{O}_{a}$ couplings in the superpotential. In this basis, each of the $X_{i}$ will have a definite anomalous dimension and a corresponding suppression factor $\epsilon_{i}$. Thus, although mixing between the $L_{i}$ will not be strictly zero in the approximately conformal regime, it will sufficiently reduced that the key approximation - that each field has its own suppression factor - will be a good one. ${ }^{2}$

For the $L_{i}$ fields in the situation just discussed, all three will have positive anomalous dimensions and all three will therefore have suppressed Yukawa couplings. However, suppose that among the operators $\mathcal{O}_{a}$ with charges conjugate to those of isospin-doublet quarks, only $\mathcal{O}_{1}$ and $\mathcal{O}_{2}$ have dimensions less than 2 . In this case only the couplings $Q_{1} \mathcal{O}_{1}$ and $Q_{2} \mathcal{O}_{2}$ can appear in the CFT; the orthogonal linear combination $Q_{3}$ will decouple, and will have no anomalous dimension in the CFT regime. The field $\bar{U}_{3}$ may similarly decouple if only two operators with appropriate charge have dimension less than 2. If both of these conditions hold, then the Yukawa coupling for $Q_{3} \bar{U}_{3}$ will be unsuppressed, and the top quark will be heavy. ${ }^{3}$ Thus, to obtain a heavy top quark while obtaining the remaining hierarchy of the standard model merely requires choosing a CFT with an appropriate spectrum of operators $\mathcal{O}_{a}$. The top quark need not be labelled in advance; the near-conformal dynamics will determine it for us.

Note that this argument would fail if the strong dynamics never becomes approximately conformal. In this case, no linear combination of the $Q_{i}$ would decouple cleanly from the CFT; the $Q_{i}$ would continue to mix. A similar fate would befall the $\bar{U}_{i}$. In this case the uptype Yukawa matrix might not have a large eigenvalue, nor would intergenerational mixing be naturally suppressed.

\section{Generic Predictions for Masses and Mixing Angles}

The main consequence of this mechanism is that low energy Yukawa coupling matrices are of the form

$$
Y_{i j}=\epsilon_{L i} \epsilon_{R j} Y_{i j}^{(0)}
$$

\footnotetext{
${ }^{2}$ Let us forestall a possible confusion. The natural basis in supersymmetric theories puts all of the renormalization into the wave function, leaving the superpotential unrenormalized. In this case one may well ask how a non-diagonal coupling matrix in the superpotential can evolve into a diagonal one! However, the physical coupling of the $X_{i}$ to the $\mathcal{O}_{a}$ is not determined by the superpotential, but is given by dressing the superpotential by the wave function matrices of $X_{i}$ and $\mathcal{O}_{a}$. These matrices can diagonalize the superpotential couplings, so that the physical interactions respect the $U(1)$ R-symmetry.

${ }^{3}$ Since the physical value of $y_{t}$ is $\sim 1$, not $4 \pi$ or $\sqrt{4 \pi}$, there is room for $y_{t}$ to be reduced a small amount from its value at $M_{0}$. Approximate, rather than strict, decoupling of the top quark from the strong sector is therefore sufficient to maintain a reasonable mass prediction.
} 
where $Y_{i j}^{(0)}$ is the short distance matrix. Assuming generic $Y_{i j}^{(0)}$, the qualitative prediction of Eq. (3.1) is that when the $\epsilon$ factors are different for different fields, then the mass matrices are approximately diagonal. These matrices are diagonalized by left- and right-handed mixing matrices with mixing angles between the $i^{\text {th }}$ and $j^{\text {th }}$ generations $\theta_{L, R_{i j}}$, of order

$$
\theta_{L i j} \sim \frac{\epsilon_{L i}}{\epsilon_{L j}} \sim \theta_{L j i}, \quad \theta_{R i j} \sim \frac{\epsilon_{R i}}{\epsilon_{R j}} \sim \theta_{R j i}, \quad(i<j)
$$

Also, the $m_{i}$ satisfy the order of magnitude relations

$$
\frac{m_{i}}{m_{j}} \sim \frac{\epsilon_{L i} \epsilon_{R i}}{\epsilon_{L j} \epsilon_{R j}}
$$

Yukawa matrices with the structure of eq. (3.1) have appeared previously in FroggattNielsen models [1] and their generic consequences are well known [1, 2, 3, 4]. In the rest of this section we summarize these predictions.

In the standard model, only the left-handed mixing angles are observable, and so we can not verify all the predictions of eqs. (3.2) and (3.3) without making additional assumptions, such as grand unification. However the generic predictions of eq. 3.2 for the CKM matrix are

$$
\begin{aligned}
V_{u d} & \sim V_{c s} \sim V_{t b} \sim \mathcal{O}(1) \\
V_{u b} & \sim V_{t d} \sim V_{c b} V_{u s} \\
V_{t s} & \sim V_{c b} \\
V_{u s} & \sim V_{c d},
\end{aligned}
$$

all of which are satisfied to within a factor of two. The last two equations follow from the first two and unitarity (if there are only three generations.) Note generational ordering is automatic: the CKM matrix is most nearly diagonal in the basis where both the up and the down-type quarks are ordered by mass.

Similarly, for neutrinos, if there is a basis in which the mixing matrix is nearly diagonal, then the lightest neutrino is mostly of the electron type and the next lightest of the muon type. In the neutrino sector we can also predict (not very stringent) order-of-magnitude bounds on $V^{L}$, the lepton analogue of the CKM matrix:

$$
\begin{aligned}
V_{e 1}^{L} & \sim V_{\mu 2}^{L} \sim V_{\tau 3}^{L} \sim \mathcal{O}(1) \\
V_{e 2}^{L} & \sim V_{\mu 1}^{L} \\
V_{\mu 3}^{L} & \sim V_{\tau 2}^{L} \\
V_{e 3}^{L} & \sim V_{\tau 1}^{L} \sim V_{e 2}^{L} V_{\tau 2}^{L} .
\end{aligned}
$$

These remain to be verified, but are allowed.

Other generic predictions of Eq. (3.3) are lower bounds on the order of magnitude of left-handed mixing angles in terms of mass ratios. These lower bounds are saturated when 
the right-handed $\epsilon$ 's are all assumed to be the same. For the CKM matrix these lower bounds are all satisfied.

$$
\begin{aligned}
& V_{u s} \gtrsim \operatorname{Max}\left(\frac{m_{d}}{m_{s}}, \frac{m_{u}}{m_{c}}\right) \\
& V_{c b} \gtrsim \operatorname{Max}\left(\frac{m_{s}}{m_{b}}, \frac{m_{c}}{m_{t}}\right) .
\end{aligned}
$$

In the lepton sector we do not know all the mixing angles or the mass ratios, but we may predict

$$
\begin{aligned}
V_{e 2}^{L} & \gtrsim \operatorname{Max}\left(\frac{m_{e}}{m_{\mu}}, \frac{m_{\nu_{1}}}{m_{\nu_{2}}}\right) \\
V_{\mu 3}^{L} & \gtrsim \operatorname{Max}\left(\frac{m_{\mu}}{m_{\tau}}, \frac{m_{\nu_{2}}}{m_{\nu_{3}}}\right)
\end{aligned}
$$

which are also allowed.

We may make more predictions for the neutrino oscillation parameters by making a reasonable assumption about the right-handed neutrinos. We assume there are at least three of these, which obtain large Majorana masses at a common scale $M$. If the only hierarchy in the right handed neutrino masses is due to suppression factors, then these will appear in both the right-handed neutrino Majorana mass matrix and in the neutrino couplings to the Higgs. After diagonalizing the neutrino mass matrix, there are three light eigenstates, which are essentially left-handed weak doublet neutrinos with small Majorana masses, and mass matrix of the form

$$
M_{\nu i j} \sim \epsilon_{L i} \epsilon_{L j} \lambda_{i a}^{(0)} \lambda_{j b}^{(0)} \epsilon_{R a} \epsilon_{R b}\left(\epsilon_{R a} \epsilon_{R b} M_{a b}^{(0)}\right)^{-1} .
$$

Note that the right-handed neutrino suppression factors $\epsilon_{R}$ cancel out in the above expression and do not affect low energy phenomenology. We can therefore predict the order of magnitude relations

$$
\begin{aligned}
\sqrt{\frac{m_{1}}{m_{2}}} & \sim V_{e 2}^{L} \\
\sqrt{\frac{m_{2}}{m_{3}}} & \sim V_{\mu 3}^{L} .
\end{aligned}
$$

These predictions are not consistent with the "just-so" solution [12, 13, 14 to the solar neutrino problem [15, 16], unless all the neutrino masses are of order $10^{-5} \mathrm{eV}$, which is inconsistent with the superKamionkande evidence for large angle $\nu_{\mu} \leftrightarrow \nu_{x}$ neutrino oscillation 17] with a mass squared difference of order $10^{-2}-10^{-3} \mathrm{eV}^{2}$. With three light Majorana neutrinos, the latter must be interpreted as nearly maximal $\nu_{\mu} \leftrightarrow \nu_{\tau}$ oscillation, and so $\epsilon_{L \mu} \sim \epsilon_{L \tau}$. If one wishes to solve the solar neutrino problem via the MSW effect [18, 19, 20], then one needs [21, 22, 23, 24, 25, 26]

$$
\frac{m_{2}^{2}}{m_{3}^{2}} \sim 10^{-2} .
$$


A mass ratio $m_{2} / m_{3} \sim 1 / 3-1 / 10$ can easily result in a seesaw model from random neutrino mass matrices; see for example ref. [27]. Thus eq. (3.19) does not require any hierarchy between $\epsilon_{2}$ and $\epsilon_{3}$, and is consistent with attributing the superKamiokande result to large angle $\nu_{\mu} \rightarrow \nu_{\tau}$ oscillations. If $\epsilon_{2} \sim \epsilon_{3}$ then the $V_{e 2}^{L}$ and $V_{e 3}^{L}$ mixing matrix elements should be of comparable magnitude. The small-angle MSW solution 24] is consistent with this, as is the CHOOZ experiment [28], if we take both angles of order .04. By contrast, the large angle MSW solution 24] is in greater difficulty, since it would require $m_{3}^{2}-m_{1}^{2} \sim m_{3}^{2}-m_{2}^{2} \gg m_{2}^{2}-m_{1}^{2}$ and $V_{e 2}^{L} \sim 1$, which is difficult to reconcile with the superKamiokande and CHOOZ experiments [28] taken together unless $V_{e 3}^{L} \lesssim 0.15$. However, as shown in ref. [27], there is actually a reasonable amount of parameter space in which an anarchic neutrino mass matrix happens to give a sufficiently small $V_{e 3}$ and large $V_{e 2}$ and $V_{\mu 3}$. We therefore do not view this scenario as clearly inconsistent with the large-angle MSW solution.

It is possible to obtain a neutrino mass hierarchy which is substantially larger than eq. (3.17). Suppose the scale at which the Majorana masses are generated is not unique, so that a hierarchy in the right-handed neutrino masses is present which is not associated with the $\epsilon$ suppression factors. This structure will generate a hierarchy of the low-energy left-handed neutrino masses which is not given by the suppression factors. However, provided that all couplings of the left-handed neutrinos are proportional to their $\epsilon$ suppression factors, then the mixing angles, and thus predictions eqs. (3.8)-(3.11) and (3.14)-(3.15), will not be affected.

\section{Generic $S U(5)$ based Predictions for Masses and Mixings}

Current available data on mass and mixing parameters hint strongly at $S U(5)$, either as a grand-unification symmetry or as an approximate symmetry of strong flavor dynamics. This has been pointed out in the context of "ten-centered" models [29, 30] and other flavor models [31, 32]. In ten-centered models, a universal suppression factor is assumed for the three $\overline{5}$ 's of $S U(5)$, and the generational hierarchy is entirely due to different suppression factors for the different 10's. These models have only four parameters, which they use to predict the nine charged fermion masses and the CKM angles. Most of the predictions are successful; two out of eight (the Cabibbo angle and the electron-to-muon mass ratio) are off by factors of 5 and $1 / 8$ respectively [29, 30]. A single additional parameter predicts the properties of the neutrino masses and mixings; in particular, large angle mixing and a small hierarchy are natural and generic in these models [29, 30, 33].

Here, we take a somewhat wider view, increasing the number of parameters by two beyond the ten-centered case, but obtaining a better fit. In particular, we allow for independent suppression factors $\epsilon_{10_{i}}$ and $\epsilon_{\overline{5}_{r}}$. Rather than assume a particular CFT, we use data to fit for the best values of the $\epsilon$ 's, and use these values to make order-of-magnitude predictions in the standard model. The predictions obtained are successful. This wider framework can more easily accommodate the small mixing angle solution to the solar neutrino problem than can the ten-centered models. It is encouraging that when one fits the suppression factors from 
the quark and lepton masses and CKM matrix, one obtains similar suppression factors for the left-handed $\mu$ and $\tau$ doublets. Thus $S U(5)$ symmetry predicts unsuppressed mixing angle for $\nu_{\mu} \leftrightarrow \nu_{\tau}$ oscillations.

The predictions of $S U(5)$ based models are (in addition to the generic predictions of the previous section):

$$
\begin{aligned}
& \sqrt{m_{c} / m_{t}} \sim V_{c b} \sim V_{t s} \sim \epsilon_{10_{2}} / \epsilon_{10_{3}} \\
& \sqrt{m_{u} / m_{c}} \sim V_{u s} \sim V_{c d} \sim \epsilon_{10_{1}} / \epsilon_{10_{2}} \\
& \frac{m_{\mu} / m_{\tau}}{\sqrt{m_{c} / m_{t}}} \sim \frac{m_{s} / m_{b}}{\sqrt{m_{c} / m_{t}}} \sim V_{\mu 3} \sim \sqrt{m_{\nu_{2}} / m_{\nu_{3}}} \sim \epsilon_{\overline{5}_{2}} / \epsilon_{\overline{5}_{3}} \\
& \frac{m_{e} / m_{\mu}}{\sqrt{m_{u} / m_{c}}} \sim \frac{m_{d} / m_{s}}{\sqrt{m_{u} / m_{c}}} \sim V_{e 2} \sim \sqrt{m_{\nu_{1}} / m_{\nu_{2}}} \sim \epsilon_{\overline{5}_{1}} / \epsilon_{\overline{5}_{2}} .
\end{aligned}
$$

Here all masses are to be assumed to be renormalized at a common, high scale.

Of course the suppression factors are somewhat uncertain, since the low-energy spectrum results from a combination of the suppression factors with numbers of order one from the assumed high-energy anarchy. Eq. (4.4) is especially ambiguous, since $m_{e} / m_{\mu}$ is smaller than $m_{d} / m_{s}$ by about a factor of 10 . If we take the geometric mean of the various determinations of mass ratios and mixing angles which go into determining the suppression factors we get the estimates:

$$
\begin{aligned}
\epsilon_{10_{2}} / \epsilon_{10_{3}} & \sim \sqrt{V_{c b} \sqrt{m_{c} / m_{t}}} \sim 0.04 \\
\epsilon_{10_{1}} / \epsilon_{10_{2}} & \sim \sqrt{V_{u s} \sqrt{m_{u} / m_{c}}} \sim 0.07 \\
\epsilon_{\overline{5}_{2}} / \epsilon_{\overline{5}_{3}} & \sim \frac{\sqrt{\left(m_{s} / m_{b}\right)\left(m_{\mu} / m_{\tau}\right)}}{\epsilon_{10_{2}} / \epsilon_{10_{3}}} \sim 0.9 \\
\epsilon_{\overline{5}_{1}} / \epsilon_{\overline{5}_{2}} & \sim \frac{\sqrt{\left(m_{d} / m_{s}\right)\left(m_{e} / m_{\mu}\right)}}{\epsilon_{10_{1}} / \epsilon_{10_{2}}} \sim 0.15 .
\end{aligned}
$$

In these expressions we have used numbers more appropriate for the GUT scale than for the weak scale, especially for $m_{t}$.

The leptonic weak mixing angles depend on the $\epsilon_{5}$ 's and are predicted to be

$$
\begin{aligned}
V_{e 2} & \sim \epsilon_{\overline{5}_{1}} / \epsilon_{\overline{5}_{2}} \sim 0.15 \\
V_{\mu 3} & \sim \epsilon_{\overline{5}_{2}} / \epsilon_{\overline{5}_{3}} \sim 0.9
\end{aligned}
$$

which is consistent with essentially maximal $\nu_{\mu} \leftrightarrow \nu_{\tau}$ oscillations and, through eq (4.3), a relatively small mass hierarchy. Eq. (4.9) lies between the expectation for either the small mixing angle or the large mixing angle MSW solution to the solar neutrino problem. This is unfortunate, since given the uncertainties from the assumed anarchy in the neutrino matrices, we cannot say that either is ruled out, or even that one is significantly favored. 


\section{Additional features: Nucleon decay and the Flavor Problem}

Another advantage of this scenario is that it suppresses nucleon decay to acceptable levels. The mechanism is analogous to the one typically observed in compositeness models, as noted in 8 , 30, 33, 34].

Even if R-parity is used to avoid dimension-four operators (here the dimension-counting refers to operators in the Lagrangian, not the superpotential) which could cause rapid nucleon decay, dimension-five operators from superpotential terms of the form $Q_{i} Q_{j} Q_{k} L_{r}$ and $\bar{U}_{i} \bar{U}_{j} \bar{D}_{r} \bar{E}_{k}$ will lead to rapid nucleon decay if these operators are suppressed only by the natural coefficient $1 / M_{p l}$. In particular, as shown in [35], the coefficients of these operators for first-generation fields cannot exceed $\left(10^{-6}-10^{-7}\right) M_{p l}^{-1}$ when first generation particles are involved.

Fortunately, in our scenario each field comes with a suppression factor related to its mass. It turns out that for moderate values of $\tan \beta$ (the ratio of the expectation values of the uptype and down-type Higgs bosons) the coefficients are sufficiently small. The decay mode $p \rightarrow K^{+} \nu$ is typically dominant. For small $\tan \beta$ we expect 36, 34 the largest contribution to come from the $Q_{2} Q_{2} Q_{1} L_{r}$ operator, along with a Cabibbo angle mixing. This gives a coefficient of order

$$
\epsilon_{10_{2}}^{2} \epsilon_{10_{1}} V_{u s} \epsilon_{\overline{5}_{r}} \sim 3 \cdot 10^{-6} \cdot \epsilon_{10_{3}}^{3} \epsilon_{\overline{5}_{r}}
$$

which requires a suppression from $\epsilon_{10_{3}}$ and/or $\epsilon_{\overline{5}_{r}}$. Note that $\epsilon_{10_{3}}$ could be as small as, say, $1 / 3$, while $\epsilon_{5_{3}}$ could be as small as $(\tan \beta / 60) \epsilon_{10_{3}}$. Thus, sufficient suppression is certainly possible, although discovery should be feasible.

However, at large $\tan \beta$ the operator $\bar{U}_{1} \bar{D}_{r} \bar{U}_{3} \bar{E}_{3}$ typically gives the dominant contribution [36, 37, 34]. This occurs through Higgsino exchange which converts the operator $u d \tilde{t} \tilde{\tau}$ to $u d s \nu_{\tau}$. The operator is proportional to $\epsilon_{10_{3}}^{2} \epsilon_{10_{1}} \epsilon_{\overline{5}_{r}}$, and the Higgsino exchange gives the Yukawa couplings $y_{t} y_{\tau}$ and a mixing angle $V_{t s}$. Altogether this gives an effective coupling of order

$$
y_{t} y_{\tau} V_{t s} \epsilon_{10_{3}}^{2} \epsilon_{10_{1}} \epsilon_{\overline{5}_{r}} \sim 10^{-4} \frac{\tan \beta}{60} \epsilon_{10_{3}}^{3} \epsilon_{\overline{5}_{r}}
$$

This dominates the previous process for large $\tan \beta$. The exact value of $\tan \beta$ where this operator produces excessive proton decay depends on many unknown parameters, such as superpartner masses, fermion mixing angles, and hadronic uncertainties. However it seems unlikely that $\tan \beta$ of order 50 , as would be needed to explain $m_{b} / m_{t}$ without suppression factors for the right handed bottom quark, can be made consistent with acceptably small proton decay. This problem is not limited to our scenario [36, 34]; many grand unified and more general flavor scenarios are in serious trouble at large $\tan \beta$ due to the absence of proton decay. (Of course particular models may escape these generic constraints [38].)

It is also generic in these models that charged-lepton branching fractions are not negligible at small $\tan \beta$ [36]. In particular, the special cancellations which occur in minimal GUTs are generally absent in these models. Since the muons and electrons which may appear come 
from the fields $L_{2}$ and $L_{1}$, we expect the ratios of their branching fractions to be of order $\left(\epsilon_{\overline{5}_{1}} / \epsilon_{\overline{5}_{2}}\right)^{2}$ [34], much larger than in typical GUTs.

In models where the CFT breaks baryon number, we expect some dimension-six baryonviolating operators in the Kähler potential to be enhanced by the CFT. This is simply because they are not inhibited by any symmetry. These operators are problematic because they are suppressed by a factor of $16 \pi^{2} / M_{c}^{2}$ and by nothing else, unlike grand unification where they are suppressed by $4 \pi \alpha_{G U T} / M_{G U T}^{2}$. This makes it clear that if baryon number is violated by strong dynamics, then $M_{c}$ cannot be far below $M_{G U T}$. If such operators do cause observable baryon number violation, the flavor structure of the branching ratios can give interesting insight into the underlying flavor physics [34]. In particular, our scenario will appear quite different from standard grand unification, since only a subset of the possible operators will be consistent with the approximate R-symmetry of the CFT.

\section{Explicit Models of Flavor}

In this section we examine several explicit examples of flavor models. We find that it is not difficult to find fairly simple supersymmetric examples of our mechanism. The main model building issues which arise are:

1. A Graceful Exit: Our mechanism requires the existence of a new gauge group and matter transforming under both new and standard gauge symmetries. It is therefore necessary for the CFT to be perturbed by small relevant operators which give mass to all colored or electrically charged states and decouple the CFT from all standard model fields at low energies. It is also important that the theory should not be driven into a phase where any standard model gauge symmetries are broken. In most cases the spectrum will include vector-like composite states carrying standard model quantum numbers. In some cases these states are parametrically lighter than $M_{c}$. Mixing between such states and the quarks and leptons may significantly reduce order of magnitude predictions for some of the Yukawa couplings. This may actually improve the agreement of the models with reality, but complicates the discussion.

2. Proton Decay: In some cases the couplings of the CFT may violate baryon number, and the graceful exit will be required to occur above $\sim 10^{15} \mathrm{GeV}$ in order to avoid proton decay from dimension-six operators in the effective Kahler potential. Obtaining a sufficiently large flavor hierarchy by then requires that the theory reaches the vicinity of a fixed point at a scale of order $\sim 10^{17} \mathrm{GeV}$ or above, and generates an anomalous dimension near 1 for some first generation particles. Avoiding proton decay from dimension-five operators places the additional constraints discussed in section 5 .

3. Landau poles: Our flavor models require a large number of new fields carrying standard gauge charges. This need not be in conflict with conventional coupling constant

unification, as explained in section 8. However it is necessary to avoid increasing the 
beta functions for standard gauge couplings too much, or they will reach a Landau pole below the quantum gravity scale. This constraint is fairly weak if most of the new matter is not much lighter than the scale of grand unification, since the gauge contribution to the beta function is large and negative above this scale. However, one must take into account that the one-loop coefficient of the standard model beta functions is nonperturbatively corrected, as we discuss in section 8. The constraint of Landau poles may be evaded if the standard model sector has a sensible Seiberg-dual description above the putative pole.

4. Acceptable Superpartner Masses: The coupling to the conformal sector has a strong effect on the masses of the scalar superpartners of the light quarks and leptons, and affects solutions of the supersymmetric flavor problem. A simple possibility is that superpartner masses are generated through low-energy gauge mediation [39]. In this case it is required that the graceful exit occurs above the messenger scale (which could be as low as $10 \mathrm{TeV}$ [40, 41]); otherwise our mechanism will undo the flavor degeneracy which is characteristic of gauge mediation. Generic supergravity models have serious flavor problems; however, in specific realizations of our flavor mechanism, these may actually be remedied by the strong dynamics. We will discuss the form of this remedy and the associated constraints on model building in section 0, and more completely in a future paper [42].

It is straightforward to build models which appear to have all of these features. However, the simplest models typically have ambiguities, such as an unknown R-charge, which makes their dynamics uncertain and limits their predictions. In some of the following models we have sacrificed elegance in favor of control and predictivity. More general models, in which the rough predictions of section 3 survive, but which put only partial constraints on the $\epsilon$ suppression factors, are both common and physically reasonable.

\subsection{A Simple Model for the Intergenerational Hierarchy based on an $S U(3)^{3}$ GUT}

In this subsection we give a simple example of a supersymmetric model which explains the intergenerational mass hierarchy. The Standard Model is embedded in the grand unified gauge group $S U(3)^{3} \otimes \mathbf{Z}_{3}$ [43, 44]. This is a subgroup of the more famous $E_{6}$ 45, with identical successful predictions for coupling constant unification. The first $S U(3)$ becomes the color group, the electroweak $S U(2)$ is embedded in the second $S U(3)$, and the hypercharge $U(1)$ is contained in both the second and third $S U(3)$ 's. The quarks and leptons are contained in three copies of the 27-dimensional representation

$$
27 \equiv(3, \overline{3}, 1)+(\overline{3}, 1,3)+(1,3, \overline{3}) .
$$

This representation also contains exotic fields; however, these are vector-like under the standard model gauge group and may obtain mass at the GUT scale.

The standard model Higgs fields, and other Higgs fields needed to break $S U(3)^{3}$ down to the standard model, may be placed in a couple of additional $27_{H}+\overline{27}_{H}$ representations. For 
arbitrary superpotential couplings, this structure results in unconstrained quark and lepton Yukawa coupling matrices to the light Higgses.

Possible Lagrangians for the Higgs sector are weakly coupled and have already been discussed in the literature [46, 47, 48, 49, 50, 51, 52, 53] so here we confine our discussion to the strongly-coupled flavor sector.

The full gauge symmetry of the model is assumed to be $S U(3)^{3} \otimes \mathbf{Z}_{3} \otimes S U(4) \otimes S U(5)$, with matter in the representation given in Table 1.

\begin{tabular}{|l|c|c|c|c|}
\hline & $S U(3)^{3}$ & $S U(4)$ & $S U(5)$ & dimension \\
\hline $27_{1}, 27_{2}, 27_{3}$ & $(3, \overline{3}, 1)+(1,3, \overline{3})+(\overline{3}, 1,3)$ & 1 & 1 & $\frac{5}{3}, \frac{4}{3}, 1$ \\
$27_{H}, 27_{H}^{\prime}$ & $(3, \overline{3}, 1)+(1,3, \overline{3})+(\overline{3}, 1,3)$ & 1 & 1 & 1,1 \\
$\overline{27}_{H}, \overline{27}_{H}^{\prime}$ & $(\overline{3}, 3,1)+(1, \overline{3}, 3)+(3,1, \overline{3})$ & 1 & 1 & 1,1 \\
$Q$ & $(3,1,1)+(1,3,1)+(1,1,3)$ & 4 & 1 & $\frac{5}{6}$ \\
$\bar{Q}$ & $(\overline{3}, 1,1)+(1, \overline{3}, 1)+(1,1, \overline{3})$ & $\overline{4}$ & 1 & $\frac{5}{6}$ \\
$Q^{\prime}$ & $(3,1,1)+(1,3,1)+(1,1,3)$ & 1 & 5 & $\frac{2}{3}$ \\
$\bar{Q}^{\prime}$ & $(\overline{3}, 1,1)+(1, \overline{3}, 1)+(1,1, \overline{3})$ & 1 & $\overline{5}$ & $\frac{2}{3}$ \\
\hline
\end{tabular}

Table 1. Quantum numbers and scaling dimensions at fixed point of chiral superfields in the model.

It has been shown [0] that $S U(4)$ and $S U(5)$ supersymmetric gauge theories with 9 flavors will flow to a superconformal phase in the infrared. The $S U(3)^{3}$ gauge groups are not asymptotically free, and their couplings will remain weak and have no effect on the dynamics. The $S U(3)^{3}$ GUT symmetry breaking is done via a conventional weakly coupled superpotential involving the various 27 's. In addition, we assume gauge-invariant and $\mathbf{Z}_{3^{-}}$ invariant superpotential couplings

$$
\sum_{i=1,2} \lambda_{i} \bar{Q} Q 27_{i}+\bar{\lambda}^{\prime} \bar{Q}^{\prime} Q^{\prime} 27_{1}
$$

Note that this is the most general gauge-invariant trilinear superpotential coupling allowed between the $Q, Q^{\prime}, \bar{Q}, \bar{Q}^{\prime}$ fields and the quarks and leptons of the three generations. Without loss of generality, the field 273 then has no relevant couplings to the superconformal sector. Since its Yukawa couplings will therefore be unsuppressed, it contains the fermions which we will identify, after the fact, with the third generation.

We describe the assumed dynamics of the theory from the top down in energy scale. First, the $S U(5)$ gauge coupling becomes strong at a scale $\Lambda_{5}$ and the theory flows to the vicinity of the infrared fixed point found by Seiberg [7]. At this fixed point, the $\bar{Q}^{\prime}, Q^{\prime}$ fields obtain anomalous dimensions of $-1 / 3$, and the $\lambda^{\prime}$ coupling becomes relevant. This coupling quickly becomes strong and we assume it drives the theory to the vicinity of a new fixed point. Here the field $27_{1}$ must acquire a positive anomalous dimension of $2 / 3$, and the coupling $\lambda_{1}$ as well as the couplings of the $27_{1}$ field to the Higgs fields become irrelevant and highly suppressed. 
The $S U(4)$ gauge coupling becomes strong at a different scale $\Lambda_{4}$ (which could be larger than $\Lambda_{5}$, but let us take it to lie near or below $\Lambda_{5}$ for the present discussion.) Below this scale the $S U(4)$ gauge dynamics are also superconformal, with the $\bar{Q}, Q$ fields acquiring anomalous dimensions of $-1 / 6$. The coupling $\lambda_{1}$ remains weak and irrelevant, but the coupling $\lambda_{2}$ is relevant and will drive the theory to a fixed point where the anomalous dimension of the field $27_{2}$ is $+1 / 3$.

For several energy decades the theory remains at this fixed point with the couplings $\lambda^{\prime}$ and $\lambda_{2}$ strong and scale invariant and all other superpotential couplings weak. A perturbative superpotential for the $27_{H}$ and $\overline{27}_{H}$ fields will break the $S U(3)^{3}$ to the standard model at a scale $M_{G} \sim 10^{16} \mathrm{GeV}$. However the $S U(3)^{3}$ remains an approximate global symmetry of all strong couplings of the theory. The scales $\Lambda_{4,5}$ could be higher or lower than $M_{G}$ without significantly affecting the analysis.

Eventually, relevant interactions drive the theory out of the superconformal phase, leaving an effective theory which is the MSSM. These interactions could most simply be mass terms $m_{4} \bar{Q} Q+m_{5} \bar{Q}^{\prime} Q^{\prime}$. However if $m_{4,5}$ are fundamental parameters it is hard to understand why they should be much smaller than the fundamental scale $M$ of the theory. A more attractive model is to add another strong group $G$, with no matter fields and gauge field strength $W$, and nonrenormalizable couplings

$$
\int d^{2} \theta \mathcal{O} \frac{1}{M^{2}} W^{2}\left(\bar{Q} Q+\bar{Q}^{\prime} Q^{\prime}\right)
$$

Condensation of the $G$ gauginos would then generate small effective mass terms for the $Q, \bar{Q}, Q^{\prime}, \bar{Q}^{\prime}$ fields and drive the theory to a phase where $S U(4)$ and $S U(5)$ are confining.

The standard-model-singlet operators $\bar{Q} Q$ and $\bar{Q}^{\prime} Q^{\prime}$ develop expectation values which break $S U(4)$ and $S U(5)$. Although these may lie above the scale $\left\langle W^{2}\right\rangle / M^{2}$, they can be arranged to lie well below the scale at which the CFT is reached, so the conformal regime is not eliminated.

The main effect of the superconformal dynamics on low energy phenomenology is to generate large wave function renormalizations for two generations of quarks and leptons. This will result in substantial suppression of all superpotential couplings for the fields of the first and second generations, with $\epsilon_{1} \sim \epsilon_{2}^{2}$ if $\Lambda_{4} \sim \Lambda_{5}$ and $m_{4} \sim m_{5}$. In this case the suppression factors are universal within a generation. Thus while the intergenerational mass hierarchy and mixing pattern are roughly explained, this theory makes the prediction that $m_{u} / m_{t}$ should be similar to $m_{d} / m_{b}$, if all Yukawa couplings are initially random. This prediction fails by about two orders of magnitude. The suppression factors also do not explain the size of $m_{b} / m_{t}$. Thus in this model not all aspects of the flavor hierarchy are explained by the renormalization group alone. Obtaining a completely realistic fermion mass spectrum is possible, however, since both mixing of standard model fields with the composites $\bar{Q} Q$ and $\bar{Q}^{\prime} Q^{\prime}$ and mixing with the exotic down-type quarks and leptons will affect the low-energy Yukawa couplings. The ratio $m_{b} / m_{t}$ could either be due to large $\tan \beta$ or to the location of the light down-type Higgs within the $27_{H}+\overline{27}_{H}$ Higgs multiplets; for instance, if the down-type Higgs were mostly a 
mixture of the $\overline{27}_{H}$ fields, its couplings to the quarks and leptons would be suppressed, since only a $27_{H}$ can couple to quarks and leptons. Detailed model building in this direction will not be attempted here.

\subsection{A "10-centered" model without proton decay}

Here we give an example of a "10-centered" model, i.e. a model which is compatible with $S U(5)$ grand unification and which produces suppression factors for two $S U(5)$ decuplets. Note that the conformal sector couplings do not induce proton decay and so $M_{c}$ may be as low as $\sim 10 \mathrm{TeV}$. Explaining $m_{b} / m_{t}$ will require either adding another conformal sector to suppress the couplings of the $\overline{5}$ 's, large $\tan \beta$, or a mechanism to suppress the couplings of $H_{d}$. The gauge group and field content of the model are listed in table 2.

\begin{tabular}{|l|c|c|c|c|}
\hline & $S U(5)_{\text {GUT }}$ & $S p(8)$ & $S p(8)^{\prime}$ & dimension \\
\hline$T_{1,2,3}$ & 10 & 1 & 1 & $\frac{42}{25}, \frac{69}{50}, 1$ \\
$\bar{F}_{1,2,3}, \bar{H}$ & $\overline{5}$ & 1 & 1 & 1 \\
$H$ & 5 & 1 & 1 & 1 \\
$Q$ & $\overline{10}$ & 8 & 1 & $\frac{87}{100}$ \\
$A$ & 1 & 27 & 1 & $\frac{3}{5}$ \\
$J, K, L, M$ & 1 & 8 & 1 & $\frac{3}{4}, \frac{3}{4}, \frac{3}{4}, \frac{9}{20}$ \\
$\bar{Q}^{\prime}$ & 10 & 1 & 8 & (confined) \\
$R, S$ & 1 & 1 & 8 & (confined) \\
\hline
\end{tabular}

Table 2. Quantum numbers and scaling dimension of chiral superfields in the 10-centered model.

We assume that a $\mathbf{Z}_{2}$ symmetry exchanges $J$ and $K$ and that the first $S p(8)$ group flows to a fixed point where the following superpotential terms are marginal:

$$
W=T_{1} Q L+T_{2} Q M+A^{5}+(J K)(J K)+A^{3} L M+(M J)(M K) .
$$

Relevant terms such as $A^{3}$ and $A^{4}$ must either be excluded by a discrete symmetry or be initially extremely small, so as not to disturb the approximate fixed point until at least the scale $M_{c}$. Since these would be forbidden by the exact R-symmetry which appears in the limit where the standard model interactions are turned off, this is technically natural. Small mass terms for $L, M$ and $A$ may be added which will drive the first $S p(8)$ away from the fixed point into a confining phase. The $S p(8)^{\prime}$ is in a confining phase as well. The composite particles are in a vector-like representation of the standard model gauge group and perturbatively irrelevant couplings between the two sectors will allow all exotic particles to get masses.

The model gives $T_{1}$ and $T_{2}$ large anomalous dimensions of $17 / 25$ and 19/50 respectively. The resulting prediction for the suppression factors

$$
\epsilon_{10_{1}}=\epsilon_{10_{2}}^{34 / 19}
$$


is in good agreement with (4.5).

\subsection{Another $S U(5)$ based example}

The following model is also consistent with $S U(5)$ grand unification, and produces suppression factors for two 10's and a $\overline{5}$ from a single $S p(12)$ gauge group at a superconformal fixed point. A $\mathbf{Z}_{2}$ symmetry (either gauged or global) is also assumed. Either large $\tan \beta$ or another sector is required to suppress the bottom quark mass. The marginal couplings of the theory do violate baryon number and will lead to proton decay from dimension-six operators in the Kahler potential. Thus an acceptable proton lifetime will require exiting the conformal regime at a scale above $10^{15} \mathrm{GeV}$. The field content of the model is given in table 3 .

\begin{tabular}{|l|c|c|c|c|}
\hline & $S U(5)_{\mathrm{GUT}}$ & $S p(12)$ & $\mathbf{Z}_{2}$ & dimension \\
\hline$T_{1,2,3}$ & 10 & 1 & 1 & $2, \frac{4}{3}, 1$ \\
$\bar{F}_{1,2,3}, \bar{H}$ & $\overline{5}$ & 1 & 1 & $\frac{5}{3}, 1,1,1$ \\
$H$ & 5 & 1 & 1 & 1 \\
$\bar{T}$ & $\overline{10}$ & 12 & 1 & $\frac{2}{3}$ \\
$A$ & 1 & 65 & 1 & $\frac{2}{3}$ \\
$F$ & 5 & 12 & 1 & 1 \\
$Z, U, V$ & 1 & 12 & $1,-1,-1$ & $\frac{1}{3}, \frac{7}{6}, \frac{7}{6}$ \\
\hline
\end{tabular}

Table 3. Quantum numbers and scaling dimension of chiral superfields

The scaling dimensions listed in the table follow from the assumption that the theory flows to a fixed point where the following superpotential terms are marginal:

$$
W=T_{1} \bar{T} Z+T_{2} \bar{T} Z A+\bar{F}_{1} F Z+\bar{T}^{3} F+\bar{T} F F Z+A U V+Z^{2} U V+Z^{2} U^{2}+Z^{2} V^{2}
$$

Small relevant mass terms for the fields $A, U$ and $V$ can eventually drive the $S p(12)$ into a confining phase. In this phase the exotic fields carrying standard model quantum numbers are in a vector-like representation and will obtain mass from the superpotential. Note that the exit is graceful. The coupling of $T_{2}$ to the CFT is removed when $A$ becomes massive, while $(T Z)$ and $(F Z)$ are both massive due to the $T F F Z$ term in the superpotential. After some order-one mixing of $T_{1}$ with $F Z$ and $\bar{F}_{1}$ with $T F$, the predictions for $T_{1}$ and $F_{1}$ are qualitatively unchanged.

\subsection{A less predictive "10-centered" model}

Here we give another example of a 10-centered model, comparable to that of section 6.2. It has the advantage of being more compact, and the disadvantage of being less predictive, although potentially just as realistic. 


\begin{tabular}{|l|c|c|c|c|}
\hline & $S U(5)_{\text {GUT }}$ & $S p(8)$ & $S p(8)^{\prime}$ & dimension \\
\hline$T_{1,2,3}$ & 10 & 1 & 1 & $?, ?, 1$ \\
$\bar{F}_{1,2,3}, \bar{H}$ & $\overline{5}$ & 1 & 1 & 1 \\
$H$ & 5 & 1 & 1 & 1 \\
$Q$ & $\overline{10}$ & 8 & 1 & $?$ \\
$L, M$ & 1 & 8 & 1 & $?, ?$ \\
$J_{1}, J_{2}, J_{3}, J_{4}, J_{5}, J_{6}$ & 1 & 8 & 1 & $?, ?, ?, ?, \frac{3}{4}, \frac{3}{4}$ \\
$\bar{Q}^{\prime}$ & 10 & 1 & 8 & (confined) \\
$\bar{J}_{1}^{\prime}, J_{2}^{\prime}$ & 1 & 1 & 8 & (confined) \\
\hline
\end{tabular}

Table 4. Quantum numbers and scaling dimension of chiral superfields in the model.

We assume that the first $S p(8)$ group flows to a fixed point where the following superpotential terms are marginal:

$$
W=\left(J_{1} J_{2}\right)^{2}+\left(J_{3} J_{4}\right)^{2}+\left(J_{5} J_{6}\right)^{2}+\left(L J_{1}\right)\left(J_{1} J_{3}\right)+T_{2} Q M+T_{1} Q L
$$

This superpotential can be assured by a gauged $\mathbf{Z}_{8}$ discrete symmetry under which $L$ and $M$ carry identical charges. The fourth term in the superpotential defines $L$, without loss of generality; the term $\left(M J_{1}\right)\left(J_{1} J_{3}\right)$ may be removed by a rotation of $L$ and $M$. The last two terms define $T_{1}$ and $T_{2}$, where we have guessed that $M$ will have larger dimension than $L$. Graceful exit may occur through masses for $J_{1}, J_{2}, J_{3}, J_{4}, L$ and $M$, after which the $S p(8)$ gauge group confines and (through small couplings such as $Q Q \bar{Q}^{\prime} \bar{Q}^{\prime}, \ldots$ ) all exotic fields become massive.

Notice that this model provides insufficient constraints to determine the R-charge of the CFT, and therefore we do not know the dimensions of most operators. However, the symmetry between $L$ and $M$ is broken, so $T_{1}$ and $T_{2}$ will have different anomalous dimensions.

This model may be altered by using other $S p$ gauge groups and changing the number of fields in the fundamental representation. It is likely that at least one of these models gives suppression factors which are consistent with data.

\subsection{Suppression factors for the $\overline{5}$ fields.}

Ideally a simple model would suppress all standard model fields at once. However, it is straightforward to suppress those of the $\overline{\mathbf{5}}$ fields separately. Let us consider a particularly simple (although not fully predictive) model. This model also is useful for illustrating another means by which a hierarchy in the suppression factors may be obtained. 


\begin{tabular}{|l|c|c|c|}
\hline & $S U(5)_{\text {GUT }}$ & $S p(4)$ & dimension \\
\hline$T_{1,2,3}, T_{0}$ & 10 & 1 & 1 \\
$\bar{F}_{1,2,3,4}, \bar{H}$ & $\overline{5}$ & 1 & $?, 1$ \\
$H$ & 5 & 1 & 1 \\
$X$ & 5 & 4 & $?$ \\
$R_{1,2,3,4,5}$ & 1 & 4 & $?$ \\
\hline
\end{tabular}

Table 5. Quantum numbers and scaling dimension of chiral superfields that lead to suppressions of $F_{i}$ fields.

The superpotential is

$$
W=\bar{F}_{i} X R_{i}+T_{0} X X
$$

One linear combination (let us call it $R_{5}$ ) of the $R_{i}$ does not couple to the $\bar{F}_{i}$. It is easy to prove that $\operatorname{dim}\left(X R_{i}\right)<2$; that $\operatorname{dim}\left(X R_{i}\right)>1$ is not proven but is extremely plausible, since the one-loop coefficient of the $S p(2)$ gauge-coupling beta function is not large. By symmetry the four $\bar{F}_{i}$ have equal and positive anomalous dimensions.

Let us assume that at some scale a dynamical mass matrix $m^{i j}$ is generated for the five fields $R_{i}$. This removes four of the $R_{i}$ and leaves one $\bar{F}_{i}$ coupled to one linear combination, call it $R_{0}$, of the $R_{i}$. Confinement of $S p(2)$ now occurs; $X R_{0}$ becomes massive with a linear combination of the $\bar{F}_{i}$, leaving three $\bar{F}_{i}$ behind to make up standard model matter. $T_{0}$ and $X X$ are also massive.

To obtain a small hierarchy in the $\epsilon_{\overline{5}_{i}}$, as in Eq. (4.5), is not difficult. Suppose that the couplings $\lambda_{i}$ in front of the $F_{i} X R_{i}$ operators are somewhat smaller than their CFT values and are slightly hierarchical at the Planck scale. They are relevant and will grow to be strong, but since $\beta\left(\lambda_{i}\right) \propto \lambda_{i}$, they will do so in a hierarchical manner, so that one, let us call it $\lambda_{1}$, might become strong before the others. In this case $\epsilon_{\overline{5}_{1}}$ will be slightly smaller than the other suppression factors, as preferred phenomenologically.

\section{The Supersymmetric Flavor Problem}

A second attractive, although not strictly necessary, feature of this scenario is that it can solve the supersymmetric flavor problem. This will be spelled out in detail in a future paper so we will only summarize the mechanism here. No such solution is necessary for gauge-mediated and some anomaly-mediated models, so let us assume we have a typical supergravity model of supersymmetry breaking, in which the contributions of the soft supersymmetry breaking terms to flavor-changing neutral currents (FCNCs) and electric dipole moments (EDMs) are generally unsuppressed. Exact relations for the renormalization of soft supersymmetry breaking terms 442, 54, 55, 56, 57, 58, 59, 60, 61] can be used to show that in our flavor scenario, the same anomalous dimensions which reduce the fermion Yukawa couplings suppress the 
trilinear scalar couplings (A-terms) between squarks/sleptons and the Higgs bosons. The A-terms therefore have a hierarchy similar to the Yukawa couplings, which reduces FCNCs and EDMs close to experimental bounds [62], provided that $\tan \beta$ is not large.

Meanwhile, as shown in [60, 63] and established generally in [42], under certain special circumstances a CFT to which neutral fields $X_{i}$ are coupled can drive the soft masses of the $X_{i}$ to zero. This happens whenever the anomalous dimension of the standard model fields can be uniquely determined from an R-symmetry contained within the superconformal algebra. For instance, this effect occurs, for all fields obtaining suppression factors, in the examples discussed in sections 6.1 6.3. Driving the soft masses of the superpartners of the light quarks and leptons to zero would be a phenomenological disaster if it occurred at the weak scale, but if it occurs at a high scale $M_{c}$, then the renormalization between the scales $M_{c}$ and $M_{W}$ will affect these masses significantly. In particular, since the gauginos are not coupled to the CFT, gaugino masses are not driven small. The gaugino masses then give positive and flavor-blind additive contributions to the light squark and slepton masses, as has been used to advantage in "no-scale" supergravity models [64, 65]. These contributions are logarithmically enhanced, and any flavor-violating effects, suppressed to near zero by the CFT, are dwarfed by the time the $\mathrm{TeV}$ scale is reached.

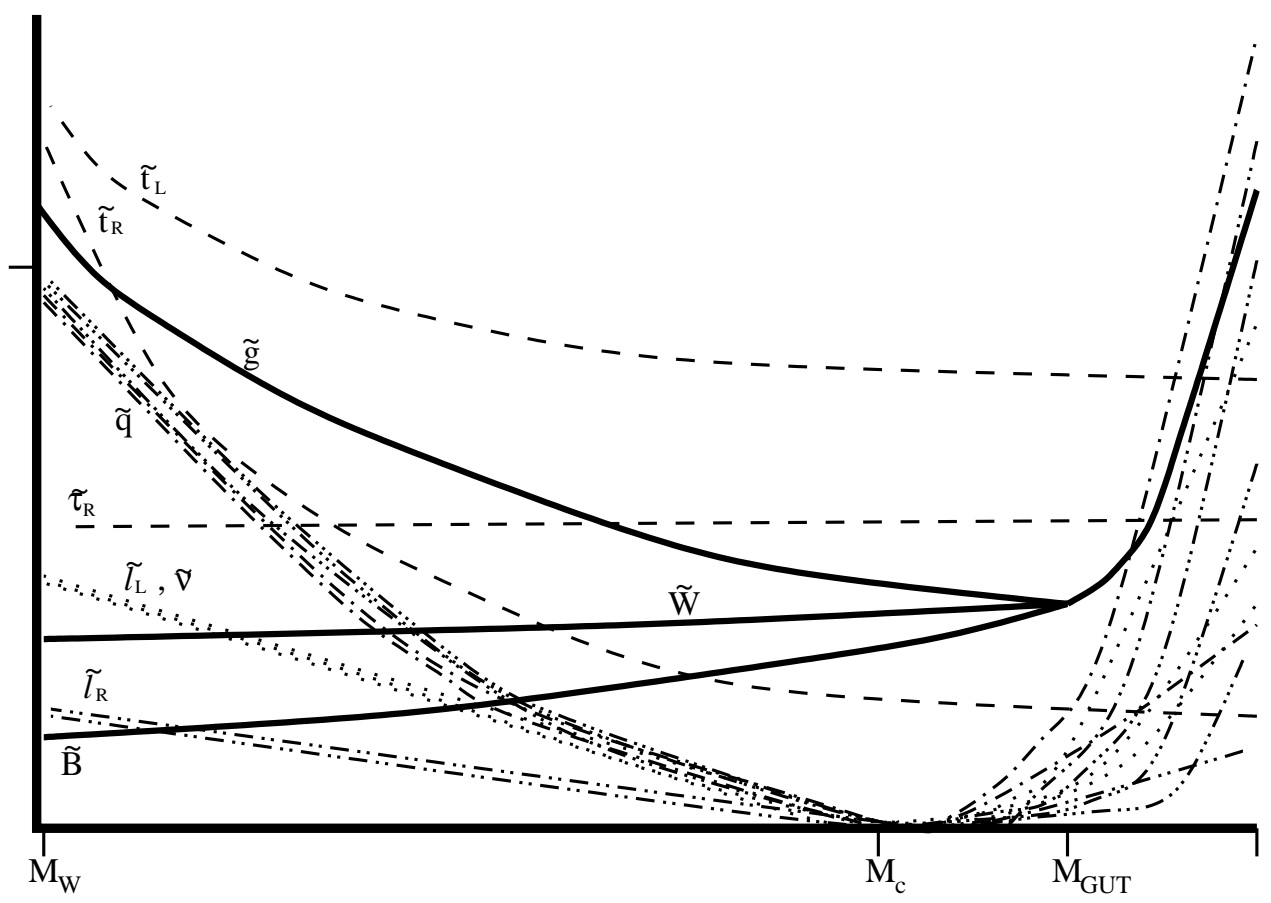

Figure 1: Schematic possible renormalization group flow for the gaugino and sfermion masses. The gauginos $\tilde{g}, \tilde{W}, \tilde{B}$ have masses which scale with the standard model gauge couplings. The sleptons $\tilde{\ell}_{L}, \tilde{\nu}$ end up degenerate, as do the $\tilde{\ell}_{R}$ sleptons and the squarks $\tilde{q}$, with the exception of some sfermions of the third generation.

Thus, the squarks and sleptons of the first two generations (and possibly the right- 
handed bottom squark and one or both tau sleptons) end up with degenerate masses; the remaining squarks and sleptons have masses which are sensitive to the Planck scale and are not predicted by the scenario. That suppression of scalar masses at a high scale ameliorates the supersymmetric flavor problem has been noted previously [66]; however our mechanism has the distinctive feature that not all scalar masses are suppressed. The qualitative behavior of the masses as a function of scale is illustrated in the figure. Quantitatively, we find that the masses at low scales are determined by a D-term for $U(1)$ hypercharge (which can be naturally generated from the soft $H_{u}$ and $H_{d}$ supersymmetry breaking masses) as well as by $M_{c}$ and by the gaugino masses. Working to one-loop in the standard model couplings, we find the first and second generation squarks have masses $\tilde{m}_{q}^{2}$ which are related to the gluino mass $M_{\tilde{g}}$ by

$$
\frac{\tilde{m}_{q}^{2}}{M_{\tilde{g}}^{2}} \approx \frac{8}{9}\left[1-\frac{\alpha_{3}\left(M_{c}\right)^{2}}{\alpha_{3}^{2}}\right]
$$

(Here and in the following, $\alpha_{i}$ with no argument is evaluated at or slightly above $M_{W}$.) Since this is not very sensitive to $M_{c}$, it is a prediction of the model that $\tilde{m}_{q} /\left|M_{\tilde{g}}\right| \lesssim .9$. The $U(1)$ D-term makes predictions for the sleptons more uncertain, but cancels out in the linear combination $\tilde{m}_{\nu}^{2}+\tilde{m}_{e^{-}}^{2}+\tilde{m}_{e^{+}}^{2}$. If we assume the gauginos have the same mass at or near the GUT scale, we find the average mass-squared for the sleptons is

$$
\frac{\tilde{m}_{\nu}^{2}+\tilde{m}_{e^{-}}^{2}+\tilde{m}_{e^{+}}^{2}}{3 M_{\tilde{g}}^{2}} \approx\left[\frac{\alpha_{2}^{2}}{\alpha_{3}^{2}}\right]\left[\frac{\alpha_{2}^{2}\left(M_{c}\right)}{\alpha_{2}^{2}}-1\right]+\frac{5}{66}\left[\frac{\alpha_{1}^{2}}{\alpha_{3}^{2}}\right]\left[\frac{\alpha_{1}^{2}\left(M_{c}\right)}{\alpha_{1}^{2}}-1\right] .
$$

This ratio is about $(1 / 4)^{2}$ if $M_{c} \sim M_{G U T}$, decreasing to $(1 / 6)^{2}$ if $M_{c} \sim 10^{11} \mathrm{GeV}$. Note that each of these two observables gives a (rather imprecise) measurement of $M_{c}$. Furthermore, at one loop the quantity $2 \tilde{m}_{q}^{2}-\frac{1}{3} \tilde{m}_{\bar{u}}^{2}-\frac{5}{3} \tilde{m}_{\bar{d}}^{2}$ is independent both of the gluino contribution and of the hypercharge D-term contribution. The hypercharge fermion loop is small, so the wino graph dominates for this observable. Numerically the left-handed squarks will be heavier than the right-handed squarks by order $30-40 \mathrm{GeV}$, if $M_{\tilde{g}} \sim 1 \mathrm{TeV}$. This gives yet another test of the model, and an independent rough measure of $M_{c}$.

Since the hypercharge gaugino has a mass of order $M_{\tilde{g}} / 6$, it cannot be the lightest supersymmetric particle (LSP) unless $M_{c}$ is around $10^{11} \mathrm{GeV}$ or above. Even for $M_{c}$ of order $M_{G U T}$, the hypercharge D-term must be positive, raising the masses of the electroweak-singlet sleptons relative to the doublet sleptons, if the the hypercharge fermion is to be the LSP. For this purpose, the D-term must be reasonably large, of order $(100 \mathrm{GeV})^{2}$.

If the slepton masses are of order $200 \mathrm{GeV}$ and those of the squarks are of order 800 $\mathrm{GeV}$, then typically the only phenomenological difficulties come from lepton-sector A-terms in the electric dipole moment of the electron and in $\mu \rightarrow e \gamma$. These are not far from the experimental bounds, however, so a small additional systematic or accidental suppression of these A terms will be enough to make the scenario viable. 


\section{Coupling Constant Unification and Fermion Mass Relations}

Any flavor model involving new strong dynamics can have an important effect on coupling constant running, potentially spoiling the coupling constant unification of the MSSM. However, as was explained in [40], coupling unification is retained if the strong dynamics respects a global symmetry into which the standard model gauge symmetry can be embedded, as long as that global group would force unification if it were gauged. Examples include $S U(5)$ or $\mathbf{Z}_{3} \times S U(3)^{3}$ global symmetries.

The proof is quite simple, and holds to one loop in the standard model couplings and to all orders in other couplings. According to 67, 68, the coupling $g_{k}$ runs as

$$
\beta_{g_{k}}=-\frac{g_{k}^{3}}{16 \pi^{2}} \frac{3 C_{2}\left(G_{k}\right)-\sum_{p} T_{k}\left(\phi_{p}\right)\left[1-\gamma_{\phi_{p}}\right]}{1-\frac{g_{k}^{2}}{8 \pi^{2}} C_{2}\left(G_{k}\right)}
$$

Here $C_{2}\left(G_{k}\right)$ is the second Casimir operator of the gauge group $G_{k}$ for which $g_{k}$ is the coupling, the sum in the numerator is over all matter fields $\phi_{p}, T_{k}\left(\phi_{p}\right)$ is half the index of the representation of $\phi_{p}$ under $G_{k}$, and $\gamma_{\phi_{p}}$ is the anomalous dimension of $\phi_{p}$. To leading order in a weak coupling constant $g_{k}$ the beta function is proportional simply to $g_{k}^{3}$ times $3 C_{2}\left(G_{k}\right)-\sum_{p} T\left(\phi_{p}\right)\left[1-\gamma_{\phi_{p}}\right]$. The usual statement of coupling constant unification is that a complete $S U(5)$ multiplet $\left\{\phi_{j}\right\}$ preserves unification because $\sum_{j} T\left(\phi_{j}\right)$ is the same for each standard model group factor, leading to equal shifts in $b_{0}=3 C_{2}\left(G_{k}\right)-\sum_{p} T\left(\phi_{p}\right)$ for the three groups and preserving both unification and the unification scale. In our case, the $S U(5)$ multiplets have large anomalous dimensions due to strong interactions involving the CFT sector. However, since the fields $\left\{\phi_{j}\right\}$ in each multiplet all have the same anomalous dimension $\gamma_{\left\{\phi_{j}\right\}}$ (by approximate $S U(5)$ flavor symmetry,) the sum $\sum_{j} T\left(\phi_{j}\right)\left[1-\gamma_{\phi_{j}}\right]=$ $\left[1-\gamma_{\left\{\phi_{j}\right\}}\right] \sum_{j} T\left(\phi_{j}\right)$ is essentially the same in each standard model group factor. Again, unification is preserved.

Note that any global symmetry which requires that

$$
\sum_{p} T_{1}\left(\phi_{p}\right) \gamma_{\phi_{p}}=\sum_{p} T_{2}\left(\phi_{p}\right) \gamma_{\phi_{p}}=\sum_{p} T_{3}\left(\phi_{p}\right) \gamma_{\phi_{p}}
$$

will make standard predictions for the unification scale and coupling constants. In addition, if $(8.2)$ is satisfied for the standard model fields alone then one can make a prediction for fermion mass relations.

The anomalous dimensions of standard model fields are related to their Higgs Yukawa couplings through

$$
\beta_{y_{i j k}}=\frac{1}{2} y_{i j k}\left[\gamma_{i}+\gamma_{j}+\gamma_{k}\right]
$$

which has solution

$$
\frac{y_{i j k}(\mu)}{y_{i j k}\left(\mu_{0}\right)}=e^{-\int\left(\gamma_{i}+\gamma_{j}+\gamma_{k}\right) d t}
$$


where $t=\ln \left(\mu_{0} / \mu\right)$. Applying the constraint (8.2) to the anomalous dimensions of the quarks and leptons gives the relation

$$
\sum_{i}\left(2 \gamma_{q_{i}}+\gamma_{\bar{u}_{i}}+\gamma_{\bar{e}_{i}}\right)=\sum_{i}\left(3 \gamma_{q_{i}}+\gamma_{\ell_{i}}\right)=\frac{6}{5} \sum_{i}\left(\frac{1}{6} \gamma_{q_{i}}+\frac{1}{2} \gamma_{\ell_{i}}+\frac{4}{3} \gamma_{\bar{u}_{i}}+\frac{1}{3} \gamma_{\bar{d}_{i}}+\gamma_{\bar{e}_{i}}\right)
$$

which implies that

$$
\sum_{i}\left(\gamma_{q_{i}}+\gamma_{\bar{d}_{i}}\right)=\sum_{i}\left(\gamma_{\ell_{i}}+\gamma_{\bar{e}_{i}}\right)
$$

If we assume that all fermion Yukawa couplings start out at the same order of magnitude, and neglect any anomalous dimensions for the Higgs, then eqs. (8.4) and (8.6) imply that, up to corrections from weak gauge couplings,

$$
\ln m_{d} m_{s} m_{b}=\ln m_{e} m_{\mu} m_{\tau}
$$

When standard model gauge corrections (principally QCD) are included then (8.7) is well satisfied.

\section{Non-supersymmetric CFT's and Flavor}

It may be possible to realize this mechanism for generating the flavor hierarchy in a nonsupersymmetric theory. Of course, such a theory must account for electroweak symmetry breaking. Since scalars in such a theory are unnatural, it is more attractive to break the electroweak symmetry dynamically, e.g. through a Higgs boson-like condensate which is a composite of two fermions [69, 70, 71]. In this case the Yukawa couplings of the fermions are in fact four-fermion couplings above the weak scale.

Here we give a brief discussion of how a flavor hierarchy might be generated, although it is not known whether the required dynamics can actually occur. Consider a theory consisting of the standard model fields and a new sector which flows to a conformal fixed point. This new sector may contain new fermion fields with four-fermion interactions between them. Note that four-fermion interactions, irrelevant if the gauge couplings are small, can be marginal or relevant when they are large, a point often used in technicolor models [72]. The ordinary quarks and leptons are assumed not to carry any non-standard gauge interactions. However, quarks and leptons may couple to three-fermion operators of this CFT. Unitarity requires the quarks and leptons to have positive anomalous dimensions, so for such couplings to be marginal or relevant the three-fermion operators must have dimension less than $5 / 2$. On the other hand, unitarity only constrains them to have dimension greater than $3 / 2$, so there is no obvious obstruction to this possibility. The quarks and leptons which couple to these operators could therefore obtain large positive anomalous dimensions. ${ }^{4}$

\footnotetext{
${ }^{4}$ This should be stated more precisely. First consider the theory in which the couplings between the quarks and leptons and the three-fermion operators are absent. Assume that the theory flows to a CFT. Now add the couplings back as small perturbations. If the dimension of the three-fermion operators is greater (less) than
} 
As in the supersymmetric case studied above, the large anomalous dimensions obtained in this way will suppress the four-fermion interactions which lead to the quark and lepton Yukawa couplings, and will thereby generate a flavor hierarchy. However, unlike the superconformal case, where certain scaling dimensions are proportional to $U(1)_{R}$ charges and are therefore additive, nonsupersymmetric theories do not have such a simple property. The scaling dimensions of products of light fermions are not the sum of the scaling dimension of those fields. Therefore, a simple relation for Yukawa matrix scaling would be guaranteed only if a separate conformal sector were introduced for each light field.

There is, however, a further dynamical concern. If quarks and leptons have only positive anomalous dimensions, then the fermion bilinear which leads to the Higgs boson would need to have scaling dimension close to unity, as in walking technicolor theories, in order to avoid suppression of the top quark mass. This could be avoided if the top quark is part of the sector which generates electroweak symmetry breaking, as in topcolor [73], or more generally, if the third generation carries any new strong gauge symmetry. If the dimension of the Higgs operator is sufficiently close to unity, then flavor physics can be pushed up to high enough scales to decouple any flavor-changing neutral current effects. Such an anomalous dimension for the Higgs operator might be natural in a large $N_{c}$ technicolor theory with matter content tuned so that the theory is nearly on the edge between the conformal and chiral symmetry breaking phases — assuming these phases actually are adjacent [74.

In strongly-coupled nonsupersymmetric conformal theories there are few known general constraints on anomalous dimensions, so we regard it to be a completely open question whether a natural and viable theory of fermion masses and electroweak symmetry breaking can be constructed without fundamental scalars or supersymmetry.

\section{Comments on String Models}

Our anarchy-suppression scenario tells a cautionary tale, and perhaps a suggestive one, for string model building. The preconditions for the scenario are natural in string theory. Yukawa couplings may easily be large, and all of the same order, at the string scale. Also, it is typical in string models for additional non-abelian gauge groups to accompany the standard model, and for there to be matter fields which are charged under both the new groups and the standard model. If all of these groups start out with moderate gauge couplings, but the standard model couplings are initially driven small due to positive beta functions, the result is a weakly-coupled standard model coupled to a strongly interacting sector. The dynamics of this new sector may be very complicated just below the compactification scale, but very often, since conformal field theories are myriad in supersymmetric models, its near-infrared

$5 / 2$, then conformal perturbation theory assures that these perturbations will be irrelevant (relevant). If the couplings are relevant the theory may flow to a new CFT. The properties of the new CFT are non-perturbative in the four-fermion couplings and cannot be inferred from the initial CFT. Thus, although there is no evidence that it is impossible, we cannot prove that the above conditions sometimes lead to a CFT with large anomalous dimensions for quarks and leptons. 
behavior will be approximately conformal. If (as is typical in string models) some standard model fields couple linearly via superpotential terms to fields charged under this new sector, they will obtain large anomalous dimensions. These will in turn have a drastic effect on the standard model Yukawa couplings to the Higgs boson, as we have described, as well as on the soft supersymmetry breaking terms.

In such models, it is completely misleading to attempt to understand flavor (and baryonnumber violation, for that matter - see section 5) using the Lagrangian obtained just below the compactification scale. Instead, one must perform a detailed field-theoretic analysis, as we have done here. In a string compactification with the standard model (or a GUT) along with an extra gauge group $G$ and extra matter $Q$, the superpotential coupling the $Q$ fields to themselves and to the standard model fields must be obtained. Then, setting all standard model gauge and Yukawa couplings to zero, one must ask whether the gauge group $G$ can have an infrared fixed point. A prerequisite for such a fixed point is an R-symmetry, anomaly free under $G$, under which all operators to have dimensions consistent with the unitarity bound. (One must approach this issue carefully, since the R-symmetry may be an accidental symmetry visible only when certain terms in the superpotential flow in the infrared to zero.) If the Rsymmetry is unique, then the dimensions of the various operators in the putative conformal field theory can be obtained and the rough effect on the standard model Yukawa couplings estimated $^{5}$. Only at this point can one determine whether the string model is potentially viable. Thereafter, one must ask the harder dynamical question of whether the conformal sector can properly decouple from the standard model without leaving over unacceptable massless matter.

\section{Conclusions}

We have demonstrated that coupling the standard model to a sector which is nearly conformal for several decades in energy can explain the striking features of the quark and lepton masses and mixing matrices. No flavor symmetry is required. This is particularly straightforward in supersymmetric theories, although the mechanism may work more generally. In the supersymmetric case, a large mass hierarchy and small mixing angles, with the largest mixing between adjacent generations, are easily understood, as is a large mixing angle for $\nu_{\mu} \leftrightarrow \nu_{\tau}$ oscillations. The well-known successful predictions of supersymmetric grand unified theories are not altered. Meanwhile, $S U(5)$-based models (which may or may not be GUTs) give successful order-of-magnitude predictions for the fermion masses, as in previously studied ten-centered models [29, 30, 33, 34]. Proton decay from dimension five operators can be naturally reduced below experimental bounds, provided $\tan \beta$ is not too large. Supersymmetric

\footnotetext{
${ }^{5}$ Even if the R-symmetry is not unique, there may still be special operators whose dimensions are known, leading to a few testable predictions in the masses and mixings of the standard model fermions. For example, the models of [75] have one-parameter families of candidate R-symmetries, but certain operators have Rcharges which are independent of the parameter. Also, unitarity constraints lead to inequalities which limit the range of the parameter. If the standard model is coupled to such a theory, the anomalous dimensions of quarks and leptons may still be well enough known to rule out many string models.
} 
flavor-changing neutral currents are greatly suppressed in a large class of models, perhaps to acceptable levels. This mechanism of suppression of flavor-changing neutral currents makes

distinctive predictions for the superpartner spectrum, which will be discussed in an upcoming paper [42].

\section{Acknowledgments}

We gratefully acknowledge the Aspen Center for Physics, where part of this work was done. M.J.S. also thanks the Institute for Theoretical Physics at the University of California Santa Barbara and the theory group at the University of Washington for their hospitality. We thank Yossi Nir for reading the manuscript and making helpful comments. A.E.N. is supported in part by DOE grant \#DE-FG03-96ER40956. The work of M.J.S. was supported by National Science Foundation grant NSF PHY95-13835 and by the W.M. Keck Foundation.

\section{References}

[1] C. D. Froggatt and H. B. Nielsen, Hierarchy of quark masses, Cabibbo angles and CP violation, Nucl. Phys. B147 (1979) 277.

[2] M. Leurer, Y. Nir, and N. Seiberg, Mass matrix models, Nucl. Phys. B398 (1993) 319-342, hep-ph/9212278.

[3] M. Leurer, Y. Nir, and N. Seiberg, Mass matrix models: The sequel, Nucl. Phys. B420 (1994) 468-504, hep-ph/9310320.

[4] Y. Grossman and Y. Nir, Lepton mass matrix models, Nucl. Phys. B448 (1995) 30-50, hep-ph/9502418.

[5] H. Georgi, A. Nelson, and A. Manohar, On the proposition that all fermions are created equal, Phys. Lett. B126 (1983) 169.

[6] T. Banks and A. Zaks, On the phase structure of vector - like gauge theories with massless fermions, Nucl. Phys. B196 (1982) 189.

[7] N. Seiberg, Electric - magnetic duality in supersymmetric nonabelian gauge theories, Nucl. Phys. B435 (1995) 129-146, hep-th/9411149.

[8] A. E. Nelson and M. J. Strassler, A realistic supersymmetric model with composite quarks, Phys. Rev. D56 (1997) 4226-4237, hep-ph/9607362].

[9] G. Mack, All unitary ray representations of the conformal group SU(2,2) with positive energy, Commun. Math. Phys. 55 (1977) 1.

[10] M. Flato and C. Fronsdal, Representations of conformal supersymmetry, Lett. Math. Phys. 8 (1984) 159.

[11] V. K. Dobrev and V. B. Petkova, All positive energy unitary irreducible representations of extended conformal supersymmetry, Phys. Lett. B162 (1985) 127.

[12] V. Barger, K. Whisnant, and R. J. N. Phillips, Realistic calculations of solar neutrino oscillations, Phys. Rev. D24 (1981) 538. 
[13] S. L. Glashow and L. M. Krauss, 'Just so' neutrino oscillations, Phys. Lett. B190 (1987) 199.

[14] A. Acker, S. Pakvasa, and J. Pantaleone, The solar neutrino problem: Some old solutions revisited, Phys. Rev. D43 (1991) 1754-1758.

[15] W. C. Haxton, The solar neutrino problem, Ann. Rev. Astron. Astrophys. 33 (1995) 459-504, hep-ph/9503430.

[16] J. N. Bahcall and M. H. Pinsonneault, Solar models with helium and heavy element diffusion, Rev. Mod. Phys. 67 (1995) 781-808, hep-ph/9505425.

[17] Super-Kamiokande Collaboration, Y. Fukuda et. al., Evidence for oscillation of atmospheric neutrinos, Phys. Rev. Lett. 81 (1998) 1562-1567, hep-ex/9807003.

[18] L. Wolfenstein, Neutrino oscillations in matter, Phys. Rev. D17 (1978) 2369.

[19] S. P. Mikheev and A. Y. Smirnov, Resonance enhancement of oscillations in matter and solar neutrino spectroscopy, Sov. J. Nucl. Phys. 42 (1985) 913-917.

[20] S. P. Mikheev and A. Y. Smirnov, Resonant amplification of neutrino oscillations in matter and solar neutrino spectroscopy, Nuovo Cim. 9C (1986) 17.

[21] G. L. Fogli, E. Lisi, and D. Montanino, A comprehensive analysis of solar, atmospheric, accelerator and reactor neutrino experiments in a hierarchical three generation scheme, Phys. Rev. D49 (1994) 3626-3642.

[22] G. L. Fogli, E. Lisi, and D. Montanino, Matter-enhanced three-flavor oscillations and the solar neutrino problem, Phys. Rev. D54 (1996) 2048-2062, hep-ph/9605273.

[23] N. Hata and P. Langacker, Solutions to the solar neutrino anomaly, Phys. Rev. D56 (1997) 6107-6116, hep-ph/9705339.

[24] J. N. Bahcall, P. I. Krastev, and A. Y. Smirnov, Is large mixing angle $M S W$ the solution of the solar neutrino problems?, Phys. Rev. D60 (1999) 093001, hep-ph/9905220.

[25] J. N. Bahcall, P. I. Krastev, and A. Y. Smirnov, Where do we stand with solar neutrino oscillations?, Phys. Rev. D58 (1998) 096016, hep-ph/9807216.

[26] G. L. Fogli, E. Lisi, D. Montanino, and A. Palazzo, Three-flavor MSW solutions of the solar neutrino problem, hep-ph/9912231.

[27] L. Hall, H. Murayama, and N. Weiner, Neutrino mass anarchy, Phys. Rev. Lett. 84 (2000) 2572, hep-ph/9911341.

[28] CHOOZ Collaboration, M. Apollonio et. al., Initial results from the CHOOZ long baseline reactor neutrino oscillation experiment, Phys. Lett. B420 (1998) 397, hep-ex/9711002.

[29] K. S. Babu and S. M. Barr, Large neutrino mixing angles in unified theories, Phys. Lett. B381 (1996) 202-208, hep-ph/9511446.

[30] M. J. Strassler, Generating a fermion mass hierarchy in a composite supersymmetric standard model, Phys. Lett. B376 (1996) 119-126, hep-ph/9510342.

[31] C. H. Albright, K. S. Babu, and S. M. Barr, A minimality condition and atmospheric neutrino oscillations, Phys. Rev. Lett. 81 (1998) 1167-1170, hep-ph/9802314. 
[32] G. Altarelli and F. Feruglio, A simple grand unification view of neutrino mixing and fermion mass matrices, Phys. Lett. B451 (1999) 388, hep-ph/9812475.

[33] M. J. Strassler, Duality in supersymmetric field theory and an application to real particle physics. Talk given at International Workshop on Perspectives of Strong Coupling Gauge Theories (SCGT 96), Nagoya, Japan. Available at http://www.eken.phys.nagoya-u.ac.jp/Scgt/proc/.

[34] K. S. Babu and M. J. Strassler, unfinished.

[35] J. Hisano, H. Murayama, and T. Yanagida, Nucleon decay in the minimal supersymmetric SU(5) grand unification, Nucl. Phys. B402 (1993) 46-84, hep-ph/9207279.

[36] K. S. Babu and M. J. Strassler, A reexamination of proton decay in supersymmetric grand unified theories, hep-ph/9808447.

[37] T. Goto and T. Nihei, Effect of RRRR dimension 5 operator on the proton decay in the minimal SU(5) SUGRA GUT model, Phys. Rev. D59 (1999) 115009, hep-ph/9808255.

[38] K. S. Babu, J. C. Pati, and F. Wilczek, Fermion masses, neutrino oscillations, and proton decay in the light of SuperKamiokande, Nucl. Phys. B566 (2000) 33, hep-ph/9812538.

[39] G. F. Giudice and R. Rattazzi, Theories with gauge-mediated supersymmetry breaking, Phys. Rept. 322 (1999) 419, hep-ph/9801271.

[40] A. Nelson and M. J. Strassler, A one-scale model of dynamical supersymmetry breaking, Phys. Rev. D60 (1999) 015004, hep-ph/9806346.

[41] H.-C. Cheng, B. A. Dobrescu, and K. T. Matchev, A chiral supersymmetric standard model, Phys. Lett. B439 (1998) 301, hep-ph/9807246.

[42] A. Nelson and M. J. Strassler, in preparation.

[43] V. A. Rizov, A gauge model of the electroweak and strong interactions based on the group SU(3)-LX SU(3)-R X SU(3)-C, Bulg. J. Phys. 8 (1981) 461.

[44] S. L. Glashow, Trinification of all elementary particle forces. Print-84-0577 (BOSTON). Published in Providence Grand Unification 1984.

[45] F. Gursey, P. Ramond, and P. Sikivie, A universal gauge theory model based on E6, Phys. Lett. B60 (1976) 177.

[46] X.-G. He and S. Pakvasa, Baryon asymmetry in SU(3)**3 x Z(3) trinification model, Phys. Lett. B173 (1986) 159.

[47] K. S. Babu, X.-G. He, and S. Pakvasa, Neutrino masses and proton decay modes in SU(3) $x$ SU(3) x SU(3) trinification, Phys. Rev. D33 (1986) 763.

[48] H. Nishimura and A. Okunishi, Strong CP problem and nucleon stability in SU(3) x SU(3) $x$ SU(3) trinification model, Phys. Lett. B209 (1988) 307.

[49] E. D. Carlson and M. Y. Wang, Trinification and the strong P problem, hep-ph/9211279.

[50] M. Y. Wang and E. D. Carlson, The breaking of the SU(3)**3 gauge group, hep-ph/9302215.

[51] G. Lazarides, C. Panagiotakopoulos, and Q. Shafi, Supersymmetric unification without proton decay, Phys. Lett. B315 (1993) 325-330, hep-ph/9306332. 
[52] G. Dvali and Q. Shafi, Gauge hierarchy in $S U(3)-c x S U(3)-L x S U(3)-R$ and low- energy implications, Phys. Lett. B326 (1994) 258-263, hep-ph/9401337.

[53] G. Lazarides and C. Panagiotakopoulos, MSSM and large tan beta from SUSY trinification, Phys. Rev. D51 (1995) 2486-2488, hep-ph/9407286.

[54] J. Hisano and M. Shifman, Exact results for soft supersymmetry breaking parameters in supersymmetric gauge theories, Phys. Rev. D56 (1997) 5475-5482, hep-ph/9705417.

[55] L. V. Avdeev, D. I. Kazakov, and I. N. Kondrashuk, Renormalizations in softly broken SUSY gauge theories, Nucl. Phys. B510 (1998) 289, hep-ph/9709397.

[56] I. Jack and D. R. T. Jones, The gaugino beta-function, Phys. Lett. B415 (1997) 383, hep-ph/9709364.

[57] I. Jack, D. R. T. Jones, and A. Pickering, Renormalisation invariance and the soft beta functions, Phys. Lett. B426 (1998) 73-77, [hep-ph/9712542].

[58] I. Jack, D. R. T. Jones, and A. Pickering, The soft scalar mass beta-function, Phys. Lett. B432 (1998) 114-119, hep-ph/9803405.

[59] A. Karch, D. Lust, and G. Zoupanos, Superconformal $N=1$ gauge theories, beta-function invariants and their behavior under Seiberg duality, Phys. Lett. B430 (1998) 254-263, hep-th/9804074.

[60] A. Karch, T. Kobayashi, J. Kubo, and G. Zoupanos, Infrared behavior of softly broken sQCD and its dual, Phys. Lett. B441 (1998) 235, hep-th/9808178.

[61] T. Kobayashi, J. Kubo, and G. Zoupanos, Further all-loop results in softly-broken supersymmetric gauge theories, Phys. Lett. B427 (1998) 291, hep-ph/9802267.

[62] A. Masiero and H. Murayama, Can epsilon'/epsilon be supersymmetric?, Phys. Rev. Lett. 83 (1999) 907-910, hep-ph/9903363.

[63] M. A. Luty and R. Rattazzi, Soft supersymmetry breaking in deformed moduli spaces, conformal theories and $N=2$ Yang-Mills theory, JHEP 11 (1999) 001, hep-th/9908085.

[64] E. Cremmer, S. Ferrara, C. Kounnas, and D. V. Nanopoulos, Naturally vanishing cosmological constant in $n=1$ supergravity, Phys. Lett. B133 (1983) 61.

[65] J. Ellis, C. Kounnas, and D. V. Nanopoulos, No scale supersymmetric GUTs, Nucl. Phys. B247 (1984) 373-395.

[66] D. Choudhury, F. Eberlein, A. Konig, J. Louis, and S. Pokorski, Constraints on nonuniversal soft terms from flavor changing neutral currents, Phys. Lett. B342 (1995) 180-188, hep-ph/9408275.

[67] M. A. Shifman and A. I. Vainshtein, Solution of the anomaly puzzle in SUSY gauge theories and the wilson operator expansion, Nucl. Phys. B277 (1986) 456.

[68] M. A. Shifman and A. I. Vainshtein, On holomorphic dependence and infrared effects in supersymmetric gauge theories, Nucl. Phys. B359 (1991) 571-580.

[69] S. Weinberg, Implications of dynamical symmetry breaking, Phys. Rev. D13 (1976) 974-996.

[70] S. Weinberg, Implications of dynamical symmetry breaking: An addendum, Phys. Rev. D19 (1979) 1277. 
[71] L. Susskind, Dynamics of spontaneous symmetry breaking in the Weinberg-Salam theory, Phys. Rev. D20 (1979) 2619.

[72] B. Holdom, Raising the sideways scale, Phys. Rev. D24 (1981) 1441.

[73] C. T. Hill, Topcolor: Top quark condensation in a gauge extension of the standard model, Phys. Lett. B266 (1991) 419-424.

[74] T. Appelquist, J. Terning, and L. C. R. Wijewardhana, The zero temperature chiral phase transition in SU(N) gauge theories, Phys. Rev. Lett. 77 (1996) 1214-1217, hep-ph/9602385.

[75] M. Berkooz, P. Cho, P. Kraus, and M. J. Strassler, Dual descriptions of SO(10) SUSY gauge theories with arbitrary numbers of spinors and vectors, Phys. Rev. D56 (1997) 7166-7182, hep-th/9705003. 